\title{
Fibrin Degradation Products in Normal and Abnormal Pregnancy and Parturition
}

\author{
JOHN BONNAR,* M.B., M.R.C.o.G.; J. F. DAVIDSON,† M.B., M.R.C.P.ED.; CHRISTINE F. PIDGEON, $\ddagger$ M.SC. \\ G. P. MCNICOL,§ M.D., PH.D., F.R.C.P.(ED., GLASG.) ; A. S. DOUGLAS,\| M.D., F.R.C.P. (LOND., ED., GLASG.)
}

\begin{abstract}
Summary : The levels of fibrin, fibrinogen degradation products (F.D.P.) in the serum were investigated in normal pregnancy and parturition, after caesarean section, and in patients with abruptio placentae, eclampsia, intrauterine death, and post-partum haemorrhage. No significant change occurred during normal pregnancy, but a highly significant increase was found during labour and again during the first week after normal delivery. After caesarean section the levels of F.D.P. were increased two to four hours after operation, and substantially higher levels were found three to eight days after operation than after normal delivery. High levels of F.D.P. were associated with abruptio placentae and eclampsia, and increased levels after intrauterine death and post-partum haemorrhage.

An excess of F.D.P. with diminished or normal systemic fibrinolytic activity suggests that local intravascular fibrin deposition and fibrinolysis occur in normal parturition and in these complications of pregnancy. The very high levels of F.D.P. found in abruptio placentae will be important in the pathogenesis of the defective haemostasis that may accompany this complication.
\end{abstract}

\section{Introduction}

The present report deals with the levels of f:brin degradation products (F.D.P.) found in normal pregnancy and parturition and in patients with complications of pregnancy ; F.D.P. levels may reflect fibrinolysis occurring in vivo in response to intravascular fibrin deposition. According to present knowledge a physiological balance between the blood coagulation and fibrinolytic mechanisms may exist which maintains an intact patent vascular tree, the coagulation system laying down fibrin on the vascular endothelium to seal any gaps which may occur and the fibrinolytic system removing such fibrinous deposits after they have served their haemostatic function. An imbalance in these mechanisms therefore might be expected in one set of circumstances-that is, diminished coagulability or excessive fibrinolysis-to lead to failure of haemostasis; alternatively, hypercoagulability and defective fibrinolysis might predispose to vascular occlusion.

In the blood coagulation system the inactive plasma protein prothrombin is converted by thromboplastin to thrombin, which in turn brings about the conversion of the soluble protein fibrinogen to insoluble fibrin. The fibrinolytic system has a similar basic structure; plasminogen, an inert plasma globulin, is converted by activators to plasmin, a proteolytic enzyme which digests fibrin and releases soluble smaller molecular weight fragments of this protein (F.D.P.). Plasminogen has a high affinity for fibrin, and when a clot

\footnotetext{
* Senior Registrar in Obstetrics and Gynaecology, Royal Maternity Hos-

pital and Victoria Infirmary, Glasgow.
+ Senior Registrar in Haematology, Royal Infirmary, Glasgow C.4.

$¥$ Research Assistant.

Senior Lecturer.

Professor of Medicine.

University Department of Medicine, Royal Infirmary, Glasgow C.4.
}

forms, enough plasminogen is incorporated to mediate the subsequent lysis of the fibrin when plasminogen activator diffuses? into the clot from the circulating plasma or surrounding endo-. thelium. After fibrin or fibrinogen is digested by plasmin $\vec{\omega}$ certain fragments are released which retain antigenic deter- $\mathscr{\omega}$ minants of the parent fibrinogen (Nussenzweig et al., 1961). $\frac{\text { J }}{3}$ Such F.D.P. are incoagulable by thrombin and hence can be found in serum ; F.D.P. are known to have pronounced anti-ci thrombin activity (Latallo et al., 1964) and to inhibit both fibrin polymerization (Fletcher, 1966) and the aggregation of platelets (Kowalski et al., 1964 ; Wilson et al., 1968); for these $\vec{\omega}$ reasons F.D.P. can produce a serious haemostatic defect. 0 Immunological methods have recently been applied to the assay of F.D.P., and the haemagglutination inhibition assay with $\overrightarrow{0}$ sensitized red cells as developed by Merskey et al. (1966) is one $\subseteq$ of the most sensitive (Murakami et al., 1965 ; Das et al., 1967).

In normal pregnancy the functioning of the coagulation and $\vec{\oplus}$ fibrinolytic mechanisms is markedly altered from that found in. the non-pregnant state, there being in pregnancy an enhanced capacity to form fibrin and at the same time reduced systemic fibrinolytic activity. The levels of F.D.P. in the circulation were therefore investigated during normal -pregnancy and labour, after operative delivery, and in patients with intrauterine $\mathbb{\mathbb { Q }}$ death, abruptio placentae, eclampsia, and post-partum $\stackrel{\Omega}{\vec{P}}$ haemorrhage.

\section{Patients and Methods}

The levels of F.D.P. were studied in 250 healthy women during uncomplicated pregnancy, labour, and the puerperium, and in 12 patients following delivery by lower uterine segment 3 caesarean section. The obstetric patients with complications consisted of 12 patients with abruptio placentae, four with $\frac{0}{3}$ intrauterine death, two with eclampsia, and four with postpartum haemorrhage. All 12 patients with abruptio placentae $\frac{}{I}$ had a moderate to severe degree of concealed retroplacental $N$ haemorrhage, as evidenced by the clinical state of the uterus and $D$ the presence of retroplacental clot at delivery. The amount of replacement transfusion in these patients varied between $2 \mathrm{~N}$ and 5 litres of blood. In the patients studied after intrauterine $N$ death in the third trimester spontaneous expulsion of the foetus $\frac{\omega}{\sigma}$ took place within 14 days $\mathrm{cf}$ the diagnosis of intrauterine death. The levels of F.D.P. in the two patients with eclampsia were determined serially following the eclamptic seizures. In the $\stackrel{\mathscr{D}}{\rightarrow}$ four patients, with post-partum haemorrhage the serious blood $\stackrel{+}{+}$ loss occurred after placental delivery and was in excess of 1 litre $\frac{0}{0}$ in each patient.

Serum F.D.P. Assay.-Venous blood samples $\left(5 \mathrm{ml}\right.$.) were $\frac{\stackrel{\rho}{\Phi}}{\Phi}$ added immediately to plastic tubes containing glass beads and $\cong$ $1 \mathrm{mg}$. of the fibrinolytic inhibitor tranexamic acid to inhibit fibrinolysis occurring in vitro. The assay method of Merskey $\frac{0}{0}$ et al. (1966) was used with minor modifications. All the serum samples were absorbed at $4^{\circ} \mathrm{C}$. for two hours with $1 / 5$ volume of packed fresh sheep red cells and then assayed immediately for F.D.P. by using the microtitre system (Cooke Engineering Co., California). Rabbit anti-human fibrinogen serum (Hoechst) was used at a dilution of $1 / 4,000$ and the citrate 
buffer for the doubling dilutions containing tranexamic acid in a concentration of $20 \mathrm{mg} . / 100 \mathrm{ml}$. Three concentrations of a fibrinogen standard, either a solution of purified human fibrinogen (Kabi) or a standard pooled plasma solution, were included in each batch of assays. The clottable protein in the standard solutions was assayed by the method of Ratnoff and Menzie (1965). The precision of the F.D.P. assay was increased by using intermediate dilutions, and the method was reproducible down to a level of $1 \mu \mathrm{g} . / \mathrm{ml}$.

In patients who had a prolonged thrombin clotting-time (McNicol and Douglas, 1964) the test serum was incubated for two hours at $37^{\circ} \mathrm{C}$. with about one-tenth of its volume of thrombin (100 units $/ \mathrm{ml}$.) to remove any residual thrombinclottable fibrinogen. In the test samples where raised levels were found the assay was repeated after further incubation with thrombin, so that so far as was possible only immunological reactive products which were not clottable by thrombin were estimated.

Plasminogen Assay.-The method of Remmert and Cohen (1949) as modified by Alkjaersig et al. (1959) was used. The method is described by McNicol and Douglas (1964).

Fibrinogen Assay.-The plasma fibrinogen levels were measured by the method of Ratnoff and Menzie (1965).

Euglobulin Lysis Time.-The test was carried out by the method described by Nilsson and Olow (1962).

Platelet Count.-A direct count was performed on venous blood as described by Dacie and Lewis (1963).

\section{Results}

The results of the assay for F.D.P. in the serum during pregnancy, labour, and the puerperium in the 250 patients are shown in Fig. 1. No significant alteration of the level took place during normal pregnancy until the first stage of labour, when the mean level rose to $3.5 \pm 2.8 \mu \mathrm{g}$. $/ \mathrm{ml}$., a highly significant rise $(t=4.555, \mathrm{P}<0.001)$. A further increase in the mean level was found during the first week of the puerperium; by the sixth week after delivery the mean serum level had returned to $1.9 \pm 1.7 \mu \mathrm{g} . / \mathrm{ml}$.

In the 12 patients studied after caesarean operations the levels of F.D.P. two to four hours after operation were in the range $1.6-20.2 \mu \mathrm{g} . / \mathrm{ml}$. (mean $7 \cdot 2 \mu \mathrm{g} . / \mathrm{ml}$.). Samples from the same 12 patients taken three to eight days after operation were in the range $1.6-108 \mu \mathrm{g} . / \mathrm{ml}$. (mean $17.9 \mu \mathrm{g} . / \mathrm{ml}$.), a significant rise $(0.01<\mathrm{P}<0.05)$. The levels of F.D.P. were thus sub-

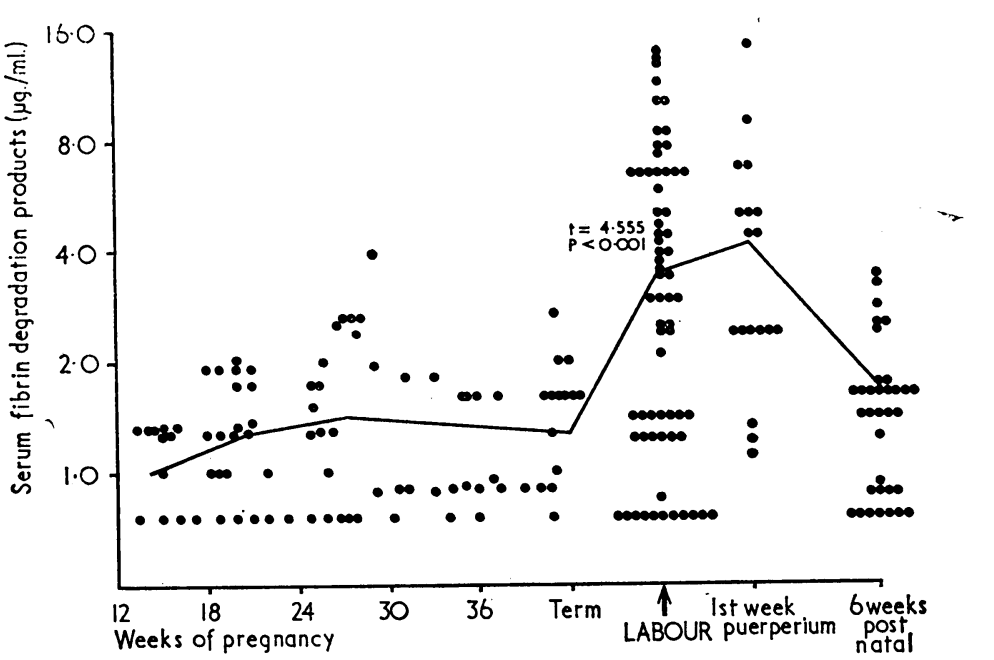

FIG. 1

FIG. 1.-Level of fibrin/fibrinogen degradation products in 250 patients during normal pregnancy, labour, and the first week after delivery (logarithmic scale). FIG. 2. - Levels of fibrin/fibrinogen degradation products (F.D.P.) in the serum of patients with abruptio placentae, (logarithmic scale). eclampsia, intrauterine death, and after post-partum haemorrhage, com pared with the mean leverithmic $x$ cale). stantially higher after caesarean operations than after normal delivery.

The levels of serum F.D.P. found in the obstetric complications studied are shown in Fig. 2, and the mean levels found in the 250 healthy women during normal pregnancy, labour, and the puerperium are included for comparison. As shown in Fig. 2 raised amounts of F.D.P. were found in each complication, and substantial levels were detected in the sera of the patients with abruptio placentae. Serial observations made in these cases of abruptio placentae showed that the level of $\bar{g}$ F.D.P. may increase rapidly during labour, but after delivery the level sharply decreased, indicating that the degradation pro- $\overline{\frac{m}{}}$ ducts are quickly eliminated from the circulation (Fig. 3). In $\widehat{\varnothing}$ the patient with abruptio placentae shown in Fig. 3 urine assay for F.D.P. when the serum level was $1,280 \mu \mathrm{g} . / \mathrm{ml}$. क showed a level of $40 \mu \mathrm{g} . / \mathrm{ml}$, suggesting that a small proportion $\vec{\circ}$ of the degradation products may be excreted in the urine. Reduced fibrinogen levels were present in all but one of the $\vec{\omega}$ patients with abruptio placentae.

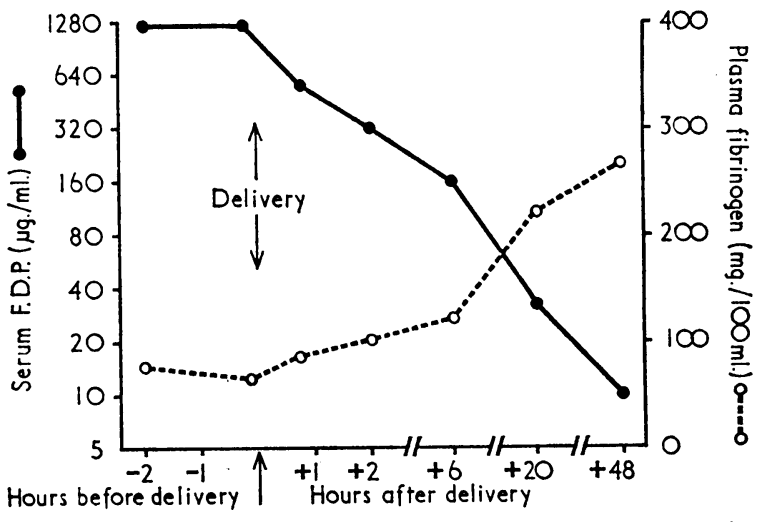

Fig. 3.-Decrease of serum fibrin/fibrinogen degradation products (F.D.P.) and spontaneous increase of plasma fibrinogen after vaginal delivery in a patient with abruptio placentae.

In the two patients with eclampsia in late pregnancy the 3 F.D.P. level in the serum rose steeply over the 48 hours after the eclamptic seizures. The findings in one of these patients are shown in Fig. 4. The euglobulin lysis time was consistently prolonged, indicating low levels of plasminogen activator in the circulating plasma; the plasminogen and fibrinogen levels were 0 also depressed ; the serum F.D.P. rose from 2.5 to $60 \mu \mathrm{g} . / \mathrm{ml} . \underline{3}$. over the four days after the eclamptic seizures, and during this period of platelet count was depressed.

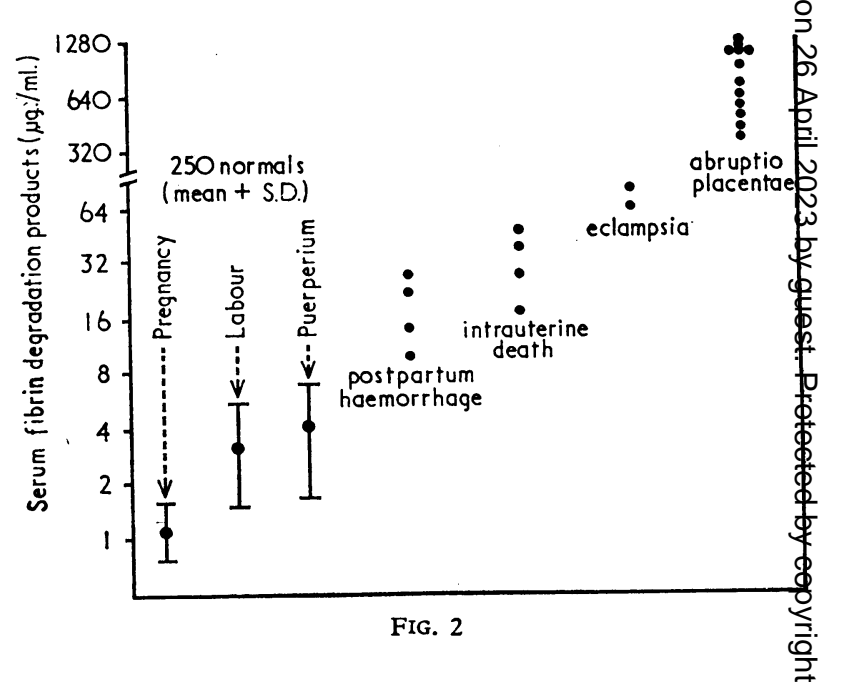


In the first two weeks after intrauterine death the level of F.D.P. was moderately raised $(8-28 \mu \mathrm{g} . / \mathrm{ml}$.) and similar levels were found in the four patients examined following postpartum haemorrhage. In both the latter groups no evidence of enhanced fibrinolytic activity was detected in the circulating plasma by the euglobulin clot lysis method.

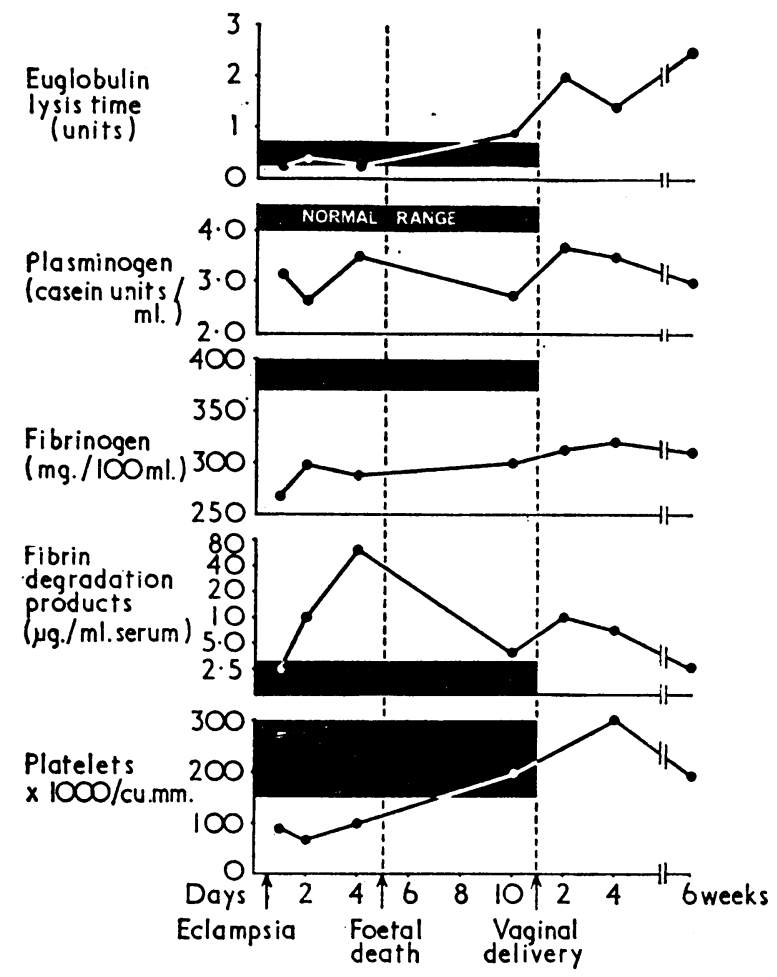

FIG. 4.-Findings in the fibrinolytic system and the platelet count after eclampsia. The normal range shown is that for normal pregnancy.

\section{Discussion}

In normal pregnancy an increased concentration of fibrinogen and the coagulation factors VII, VIII, and X has been reported (Pechet and Alexander, 1961 ; Talbert and Langdell, 1964 ; Nilsson and Kullander, 1967), and in late pregnancy and labour a marked reduction of fibrinolytic activity has been found (Bonnar et al., 1969). The significance of these changes in normal pregnancy is uncertain, but the haemostatic mechanism would appear to be altered towards a physiological hypercoagulability with an enhanced capacity to form fibrin and a diminution in the ability to lyse fibrin. The immunological assay used in this study of circulating F.D.P. does not distinguish between the degradation products of fibrin and those of fibrinogen. It is of interest that no significant alteration of the level of degradation products could be shown during pregnancy despite the increase of plasma fibrinogen. A recent study by Woodfield et al. (1968) reported progressively higher levels of degradation products in the second and third trimesters, but we found no significant increase of the level until the onset of labour.

The sharp increase in the levels of degradation products during labour is reliable evidence that active fibrinolysis is taking place. In the presence of diminished fibrinolytic activity in the circulation, a striking and consistent finding in labour, the increased levels of degradation products in the serum may be explained by the lysis of fibrin in localized areas of the vascular compartment. Fibrin plaques and numerous intervillous thrombi are found on the maternal side of the majority of normal placentae (Fox, 1963 ; Devi et al., 1968). The uterus is a potent source of plasminogen activator (Albrechtsen, 1957 ;
MacKay et al., 1967), and uterine action during labour is accompanied by transient myometrial ischaemia. Circumstances therefore may exist for the local release of activator, and this may then be absorbed on placental fibrin with consequent local fibrinolytic activity in the placental circulation. An enhanced fibrinolytic activity in the uterine circulation during labou could be a natural defence mechanism to maintain the maternas blood supply to the placenta by ensuring the patency of the placental bed during labour.

The raised levels of F.D.P. in the puerperium after normat delivery are possibly related to the removal of fibrin laid dowe to secure haemostasis in the uterus after separation of the placenta. The higher levels found after delivery by caesarea $\bar{D}$ section may possibly reflect the more extensive tissue damage associated with operative delivery.

With the exception of one patient with abruptio placenta $\vec{e}$ who had transient evidence of a proteolytic state the high level $\vec{E}$ of F.D.P. in abruptio placentae and after eclampsia were preseng in association with greatly diminished lytic activity in the circulation. This suggests that the abnormal amounts of degradation products in the serum are most likely the result of localized lysis of fibrin in the vascular compartment rather than proteolysis of circulating fibrinogen. The raised levels of F.D.P. may therefore indicate that in these complications of pregnancy both intravascular fibrin formation and fibrito proteolysis are taking place.

The placenta and decidua were observed by Seegers an $\overrightarrow{c 0}$ Schneider (1951) to be particularly rich in clot-promoting sub stance, and in pregnant women dying soon after abruptio placentae emboli of fibrin-like material have been found in the pulmonary arterioles and in other organs (Schneider, 1951, Mayer et al., 1954 ; Johnstone and McCallum, 1956 ; Beischer 1961). In animal experiments Brown and Stalker (1968) demonstrated that after induced premature separation of the placent white emboli resembling fibrin emboli were released from theू placental bed into the maternal and foetal circulations. The activation of the plasminogen system is therefore likely to be direct or indirect protective response to intravascular clotting 3 High levels of degradation products of fibrin would also be in accord with the concept that lysis of intravascular fibrin is due to local activation of plasminogen within the thrombi (Sherry et al., 1959). The reduced levels of plasminogen activator in pregnancy (Shaper et al., 1965 ; Nilsson and Kullander 1967 ; Bonnar et al., 1969) may be important in the patho-5 genesis of such conditions as acute tubular necrosis in association with severe placental abruption, as low levels of activator could delay the removal of intravascular fibrin in the renal circulation; animal studies have shown that the level of plasminogen activator in the endothelium of renal vessels is decreased during pregnancy (Epstein, 1966).

High levels of F.D.P. are of importance in relation to the defective haemostasis which may present in abruptio placentae $N$ as the degradation products can interfere with normal fibrin polymerization, the thrombin-fibrinogen reaction, and the func- tion of the platelets, with the result that clot formation may be delayed and defective. The rapid decrease of the level of circulating F.D.P. after delivery emphasizes that prompt vaginat? delivery without trauma is likely to be the most effective wayo of restoring the haemostatic process to normal.

In the two patients with eclampsia the level of degradation products rose steeply over the four days after the eclamptic seizures. The rise in levels of degradation products in association with depression of the platelet count and the fibrinogeno and plasminogen levels suggests that an episode of intravascularo coagulation may have taken place. Increased fibrinolytic activity has been found in animals after electrically induced convulsions (Fantl and Simon, 1948) and in patients following electroshock therapy (Fletcher et al., 1963); it is of interest that following the convulsions of eclampsia high levels of F.D.P. appeared but systemic fibrinolytic activity remained 
markedly depressed. Immunofluorescence studies on the renal glomerulus in pre-eclampsia have shown that the glomerulus contains fibrin, an observation which has been confirmed by electron microscopy (McKay, 1966). McKay (1965) has postulated that in pre-eclampsia low-grade intravascular coagulation is present and that the convulsions are the result of sudden agglutination of platelets and the formation of platelet and fibrin thrombi which obstruct the cerebral microcirculation. The findings in the two patients currently presented would be consistent with such a course of events.

Foetal death in utero is followed by a gradual depletion of clotting factors (Pritchard and Ratnoff, 1955). Intravascular coagulation has been widely incriminated as the usual cause of fibrinogen depletion in patients with a retained dead foetus (Pritchard, 1959 ; Hardisty and Ingram, 1965). The report of Lerner et al. (1967) that the depleted coagulation factors after intrauterine death were corrected by the administration of heparin alone, without blood or fibrinogen, and our findings of raised serum F.D.P. after intrauterine death are strong evidence that intravascular coagulation is the mechanism responsible for the depletion of the clotting factors.

The levels of F.D.P. were only slightly raised in the patients with post-partum haemorrhage, in contrast to the patients with abruptio placentae, in whom very high levels were found. Hardaway (1966) has shown experimentally in dogs that haemorrhagic shock is associated with intravascular clotting if hypotension occurs in association with circumstances which favour clotting, such as high levels of coagulation factors. The presence of fibrin thrombi in the pituitary was noted by Sheehan and Murdoch (1938) in their extensive report on necrosis of the anterior pituitary in patients dying after haemorrhagic collapse at delivery. The rapid restoration of the blood volume and correction of hypotension probably limits the progression of intravascular fibrin formation and may explain the rarity of Sheehan's syndrome following post-partum haemorrhage in modern obstetric practice.

The demonstration in this investigation of abnormal amounts of fibrinolytic degradation products suggests that intravascular fibrin deposition and fibrinolysis are occurring in varying degrees in normal parturition and in abruptio placentae, intrauterine death, eclampsia, and post-partum haemorrhage. The clinical outcome in these complications of pregnancy probably depends on the balance between clotting and lysis as the condition evolves, but both thrombotic vascular occlusion and defective haemostasis are possible sequelae.

We are grateful to Sir Hector MacLennan and Professor E. M. McGirr for their interest in this work, and to the consultant staff of Glasgow Royal Maternity Hospital for their co-operation in this investigation; to the Medical Research Council and to F.B.A. Pharmaceuticals Ltd. for financial assistance ; and to Miss P. O'Neil for laboratory assistance.

John Bonnar is joint holder of the Samuel Research Scholarship awarded by the Royal College of Obstetricians and Gynaecologists.

\section{REFERENCES}

Albrechtsen, O. K. (1957). British fournal of Haematology, 3, 284. Alkjaersig, N., Fletcher, A. P., and Sherry, S. (1959). Fournal of Clinical Investigation, 38, 1086

Beischer, N. A. (1961). American fournal of Obstetrics and Gynecology, 82, 625 .
Bonnar, J., McNicol, G. P., and Douglas, A. S. (1969). British Medical fournal. In press.

Brown, C. J., and Stalker, A. L. (1968). Proceedings of the International Conference on Microcirculation, Gothenburg, Sweden, 1968. Gothenburg, Sweden.

Dacie, J. V., and Lewis, S. M. (1963). Practical Haematology, 3rd ed., p. 61. London, Churchill.

Das, P. C., Allan, A. G. E., Woodfield, D. G., and Cash, J. D. (1967) British Medical fournal, 4, 718

Devi, B., Jennison, R. F., and Langley, F. A. (1968). Fournal of Clinical Pathology, 21, 322.

Epstein, M. D. (1966). Federation Proceedings, 25, 620.

Fantl, P., and Simon, S. E. (1948). Australian fournal of Experimental Biology and Medical Science, 26, 521.

Fletcher, A. P. (1966). Federation Proceedings, 25, 84.

Fletcher, A. P., Biederman, O., Moore, D., Alkjaersig, N., and Sherry, S. (1963). Transactions of the Association of American Physicians, 76, 280.

Fox, H. (1963). Fournal of Obstetrics and Gynaecology of the British Commonwealth, 70, 980 .

Hardaway, R. M. (1966). Syndromes of Disseminated Intravascular Coagulation, p. 5. Illinois, Thomas.

Hardisty, R. M., and Ingram, G. I. C. (1965). Bleeding Disorders, p. 108. Oxford, Blackwell Scientific Publications.

Johnstone, J. M., and McCallum, H. M. (1956). Scottish Medical fournal, 1,360 .

Kowalski, E., Budzynski, A. Z., Kopec, M., Latallo, Z. S., Lipinski, B., and Wegrzynowicz, Z. (1964). Thrombosis et Diathesis Haemorrhagica, 12, 69.

Latallo, Z. S., Budzynski, A. Z., Lipinski, B., and Kowalski, E. (1964) Nature, 203, 1184.

Lerner, R., Margolin, M., Slate, W. G., and Rosenfeld, H. (1967). American fournal of Obstetrics and Gynecology, 97, 373.

MacKay, A. V. P., Das, P. C., Myerscough, P. R., and Cash, J. D. (1967). Fournal of Clinical Pathology, 20, 227.

McKay, D. G. (1965). Disseminated Intravascular Coagulation, p. 124. New York, Hoeber.

McKay, D. G. (1966). In Coagulation Disorders in Obstetrics, edited by J. C. de Neef and G. J. H. den Ottolander. Amsterdam, Excerpta Medica Foundation.

McNicol, G. P., and Douglas, A. S. (1964). In Recent Advances in Clinical Pathology, Series 4, edited by S. C. Dyke, p. 187. London, Churchill.

Mayer, M., Dreyfus, C., and Bugnard, C. (1954). Gynaecologia, 138, 87

Merskey, C., Kleiner, G. J., and Johnson, A. J. (1966). Blood, 28, 1.

Murakami, M., et al. (1965). Fapanese fournal of Clinical Pathology, 13, 542.

Nilsson, I. M., and Kullander, S. (1967). Acta Obstetrica et Gynecologia Scandinavica, 46, 273.

Nilsson, I. M., and Olow, B. (1962). Acta Chirurgica Scandinavica, 123, 247

Nussenzweig, V., Seligmann, M., Pelmont, J., and Grabar, P. (1961) Annales Institut de Pasteur, 100, 377.

Pechet, L., and Alexander, B. (1961). New England fournal of Medicine, $265,1093$.

Pritchard, J. A. (1959). Obstetrics and Gynecology, 14, 573.

Pritchard, J. A., and Ratnoff, O. D. (1955). Surgery, Gynecology and Obstetrics, 101, 467.

Ratnoff, O. D., and Menzie, C. (1965). In Blood Coagulation, Hemor-

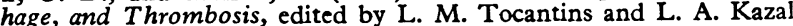
p. 224. New York, Grune and Stratton.

Remmert, L. F., and Cohen, P. P. (1949). Fournal of Biological Chem istry, 181, 431.

Schneider, C. L. (1951). Surgery, Gynecology and Obstetrics, 92, 27.

Seegers, W. H. and Schneider, C. L. (1951). American fournal of Obstetrics and Gynecology, 61, Suppl., p. 469.

Shaper, A. G., Macintosh, D. M., Evans, C. M., and Kyobe, J. (1965). Lancet, 2, 706.

Sheehan, H. L., and Murdoch, R. (1938). Fournal of Obstetrics and Gynaecology of the British Empire, 45, 456.

Sherry, S. Fletcher A. P., and Alkjaersig, N. (1959). Physiological Reviews, 39, 343 .

Talbert, L. M., and Langdell, R. D. (1964). American fournal of Obstetrics and Gynecology, 90, 44.

Wilson, P. A., McNicol, G. P., and Douglas, A. S. (1968). Fournal of Clinical Pathology, 21, 147.

Woodfield, D. G., Cole, S. K., Allan, A. G. E., and Cash, J. D. (1968). British Medical fournal, 4, 665. 